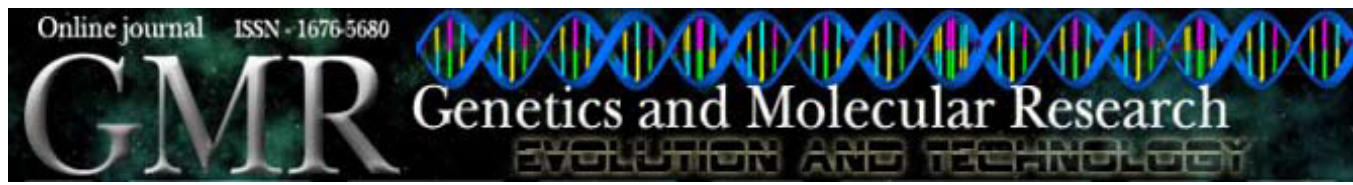

$\underline{\text { Thesis Abstract }}$

\title{
Characterization of the nucleolar cycle and chromatoid body formation in the spermatogenesis of some vertebrate species
}

\author{
R.L. Peruquetti \\ 2009. Pós-graduação em Genética, Instituto de Biociências, Letras e \\ Ciências Exatas, UNESP/IBILCE, São José do Rio Preto, SP, Brasil. \\ Ph.D. thesis. Orienting Professor: Dr. Maria Tercília Vilela de \\ Azeredo Oliveira
}

The chromatoid body $(\mathrm{CB})$ is a cytoplasmic organelle that has a function related to RNA and protein accumulation and/or storage for later germ-cell differentiation. Many theories have been postulated to explain the origins of the $\mathrm{CB}$ material. One of the most accepted theories indicates that $\mathrm{CB}$ originates from nucleolar material, in which it was fragmented during early spermatogenesis; this fragmented nucleolar material migrates to the cytoplasm. Our objectives were to: 1) monitor nucleolar material distribution by cytochemical techniques (hematoxylin-eosin, toluidine blue, modified critical electrolyte concentration for detecting RNA, silver-ion impregnation, and Feulgen reaction) and by ultrastructural analysis (transmission electron microscopy), and 2) compare nucleolar material distribution with the formation of $\mathrm{CB}$ in some vertebrate species: Tilapia rendalli (Teleostei, Cichlidae); Dendropsophus minutus (Amphibia, Anura); Phrynops geoffroanus (Reptilia, Testudines), and Oryctolagus cuniculus (Mammalia, Lagomorpha). We found that nucleolar fragmentation occurred during the beginning of prophase I and nucleolus reorganization occurred in the early spermatid nucleus. The area of the early spermatid nucleolus was significantly smaller than that of the spermatogonium nucleolus. Three phenomena could contribute to the difference between the spermatogonium nucleolar area and the early spermatid nucleolar area: a) modification of cell activity; b) decrease in the number of nucleolus organizer regions in the spermatids, and c) migration of fragmented nucleolar material from the nucleus to the cytoplasm. This nucleolar material will participate in the $\mathrm{CB}$ formation process. Ultrastructural analysis showed an accumulation of nuages, which form the $\mathrm{CB}$ before nucleolar fragmentation, in the spermatogonium cytoplasm of $T$. rendalli and $D$. minutus. Otherwise, the CB in $P$. geoffroanus and $O$. cuniculus is formed only in the primary spermatocyte cytoplasm, when the nucleolus was fragmented. The CB was found to be associated with mitochondrial clusters in the primary spermatocyte cytoplasm. This cytoplasmic organelle was observed in associa- 
tion with the Golgi complex in the primary spermatocytes of $P$. geoffroanus. This unique cloud-like structure of male germ cells was detected near the region of acrosomal vesicle formation; it was associated with mitochondria in the early spermatids of $P$. geoffroanus and $O$. cuniculus. This association was absent in the early spermatids of $T$. rendalli and $D$. minutus. The CB was linked with some lipid droplets in the early spermatid cytoplasm of P. geoffroanus. The later spermatids in all species had CB near the region where formation of the mitochondrial sheath and spermatozoon tail took place, which suggests that $\mathrm{CB}$ is related to the development of these structures. In conclusion, the nucleolus is fragmented at the beginning of prophase I. This fragmented nucleolar material probably migrates to the cytoplasm, where it will participate in the formation of the CB. The CB seems to have important functions in the spermatogenesis of some vertebrate species. 